\title{
Genetic Differentiation of Magnaporthe oryzae Populations from Scouting Plots and Commercial Rice Fields in Korea
}

\author{
S.-Y. Park, M. G. Milgroom, S. S. Han, S. Kang, and Y.-H. Lee
}

First and fifth authors: Department of Agricultural Biotechnology, Center for Fungal Genetic Resources, and Center for Agricultural Biomaterials, Seoul National University, Seoul 151-921, Korea; second author: Department of Plant Pathology, Cornell University, Ithaca, NY 14853; third author: National Institute of Crop Science, Rural Development Administration, Suwon 441-857, Korea; and fourth author: Department of Plant Pathology, The Pennsylvania State University, University Park 16802.

Accepted for publication 7 December 2007.

\begin{abstract}
Park, S.-Y., Milgroom, M. G., Han, S. S., Kang, S., and Lee, Y.-H. 2008. Genetic differentiation of Magnaporthe oryzae populations from scouting plots and commercial rice fields in Korea. Phytopathology 98:436-442.

A previous study of the diversity and population structure of the rice blast fungus, Magnaporthe oryzae, over a 20-year period in Korea, found novel fingerprint haplotypes each year, and the authors hypothesized that populations might experience annual bottlenecks. Based on this model, we predicted that $M$. oryzae populations would have little or no genetic differentiation among geographic regions because rice blast is commonly found throughout Korea each year and $M$. oryzae would have to disperse from small populations surviving annually between rice crops. To test this hypothesis, we sampled $M$. oryzae from rice fields in eight provinces in Korea in a single year (1999). In four provinces, we sampled from a set of rice cultivars commonly grown in commercial fields (group I); because of low disease incidence in four other provinces, we could not sample from commercial fields and instead sampled from scouting plots of different cultivars set up for detecting new pathotypes of M. oryzae (group II). All

and II), with haplotype similarities of $71 \%$ between lineages and $>76 \%$ within lineages. Isolates from the same cultivar within group I were genetically differentiated among locations, and isolates within the same location were differentiated among cultivars. Differentiation for $T L H 1$ and $P W L 2$ was significant $(P<0.03)$, but not as strong as for fingerprint markers. Similar analyses were not possible among group II isolates because too few isolates were available from any one cultivar. All isolates were in the same mating type, Mat1-1, ruling out sexual reproduction as a source of novel haplotypes. When the 1999 samples were compared with the historical samples from the previous study, haplotypes of group I formed a separate cluster, while those of group II clustered with haplotypes from the historical sample. Altogether, geographic subdivision, monomorphism of mating type, and correlation of haplotypes to sets of cultivars are not consistent with the hypothesis of repeated turnover of haplotypes. Instead, the previous correlations of haplotypes to year might have been caused by inadequate sampling of haplotypes each year, highlighting the need for studies of population genetics to be conducted with systematic samples collected to address specific questions.
\end{abstract} isolates were genotyped with DNA fingerprint probes MGR586 and MAGGY, a telomere-linked gene family member $T L H 1$, the $P W L 2$ host specificity gene and mating type. Fingerprint haplotypes clustered into two distinct lineages corresponding to the two sets of cultivars (groups I
Additional keywords: bottleneck, genetic drift, Magnaporthe grisea, Pyricularia oryzae, rice blast.

tions. Although $M$. oryzae seems to be nearly genetically homogeneous at the nucleotide level worldwide $(2,3)$, analyses of geographic structuring using more rapidly evolving genetic markers, such as DNA fingerprint markers that can discriminate among asexual lineages, have been more informative. Some studies have described the distribution of lineages among different locations. For example, Kumar et al. (12) found some of the same lineages in Himalayan valleys $100 \mathrm{~km}$ apart, separated by mountains; they also found some lineages in the Himalayan foothills and $1,000 \mathrm{~km}$ away in lowland plains in India. From both of these comparisons, they concluded that $M$. oryzae had migrated between populations. Other studies, conducted on limited geographic areas, showed the presence of clonal lineages in multiple areas, suggesting a widespread migration of $M$. oryzae $(10,17,22)$. One of the most detailed studies of geographic (and temporal) structure of $M$. oryzae populations was done by Chen et al. (1) comparing samples from upland and lowland rice-growing areas in the Philippines. Although they sampled from the same cultivars, they found significant genetic differentiation between the two sites. However, the upland site was planted mainly to tropical japonica varieties used in breeding trials and was geographically isolated from commercial rice production. The lowland site, in contrast, was also in an experimental breeding field, but was surrounded by commercial rice production of indica varieties. Therefore, geographic locations were confounded 
with differences in ecological conditions and surrounding host populations.

In a previous study (16), using isolates collected over nearly two decades, we evaluated the population structure and dynamics of $M$. oryzae in Korea with respect to pathotypes and DNA fingerprint haplotypes. DNA fingerprinting analyses of 176 isolates that were randomly chosen from strain collections made from 1984 to 1999 for pathotype surveys showed no clear lineage structure, and no correlation between pathotypes and fingerprint similarity. These results suggest that $M$. oryzae populations in Korea have been dominated by a single clonal lineage with diverse pathotypes, similar to the population in Japan (4), where only two lineages were detected. Contrary to most other studies, we did not find any evidence in Korea for the host population selecting for specific sets of pathotypes.

Although we did not find a clear lineage structure or correlation between hosts and fingerprints in our previous study in Korea (16), we detected a correlation between the year of collection and haplotype similarity. For example, we found two to four subgroups (or sublineages within the single lineage) in most years, although some years had only one. With a few exceptions, the same subgroup was not found in two successive years, implying that there is a turnover of fingerprint haplotypes between years. We speculated (16) that this correlation could arise if populations of $M$. oryzae undergo annual bottlenecks in which relatively few haplotypes survive from one rice-growing season to the next, such that isolates sampled each year are derived from a few founder individuals, and variation within subgroups accumulates due to mutation. This hypothesis has significant epidemiological implications because it suggests that the rice blast pathogen does not survive in large numbers between crops. However, blast occurs in most rice-growing areas in Korea each year, even though incidence is variable, depending on environmental conditions (unpublished data). If $M$. oryzae undergoes annual bottlenecks and blast is widespread each year in Korea, the few individuals that persist between years must disperse throughout large areas during each cropping season. Based on this model, we predicted that we would find little evidence for genetic differentiation among subpopulations from different regions in Korea, similar to the widespread occurrence of fingerprint lineages and lack of differentiation observed in other populations $(10,12,17,22)$.

In addition to repeated bottlenecks, two other hypotheses could explain the correlation between fingerprint similarity and year observed previously. First, selection for different pathotypes because of temporal variation in rice cultivars could potentially alter the composition of the $M$. oryzae population. However, a small subset of cultivars consistently dominated rice cultivation in Korea during the course of the study, and we did not detect any obvious correlation between fingerprint subgroups and pathotypes or host origins (16). Second, sexual reproduction of $M$. oryzae could produce novel haplotypes; survival of a small sample of these (a bottleneck), followed by asexual reproduction, could result in a few genetically distinct haplotypes. This hypothesis would explain the occurrence of novel haplotypes, and coupled with bottlenecks, could explain the relatively few subgroups observed each year.

To address the underlying causes of correlations between haplotype similarity and year of sampling, we needed a different sampling strategy than what was used previously. Therefore, the overall objective of this study was to analyze the genetic structure of the M. oryzae population in Korea in a single year, using larger sample sizes and more structured sampling with respect to locations. We had the following specific objectives: (i) to estimate genetic differentiation among $M$. oryzae subpopulations in eight provinces in Korea; (ii) to look for correlations of fingerprint haplotypes to rice cultivars as evidence of selection by the host population; and (iii) to determine the mating-type ratios of M. oryzae in Korea, as a way of assessing the potential for sexual reproduction.

\section{MATERIALS AND METHODS}

Sampling and isolation of $M$. oryzae. Sampling of M. oryzae was done during the rice-growing season of 1999 using two strategies. Our original intention was to sample $M$. oryzae from all eight provinces of Korea (Fig. 1), from a subset of nine common cultivars (Table 1). However, because of low disease incidence in some areas, we obtained 180 isolates from these cultivars in only four provinces (Kyonggi, Kangwon, Chonnam, and Chonbuk). Isolates of $M$. oryzae were collected from one rice field in each province, except for Kangwon where we sampled from two fields, $85 \mathrm{~km}$ apart. Cultivars present in each field are shown in Table 1. These isolates are referred to as group I.

To sample from the four remaining provinces (Kyongnam, Kyongbuk, Chungnam, and Chungbuk), we obtained 74 isolates from the Rural Development Administration (RDA) of Korea, which conducts annual pathotype surveys of $M$. oryzae. The agroecological conditions in these four provinces are not substantially different from the other provinces even though less rice blast was found in 1999. Isolates from this survey (referred to as group II) were collected from a large number of cultivars in a total of 26

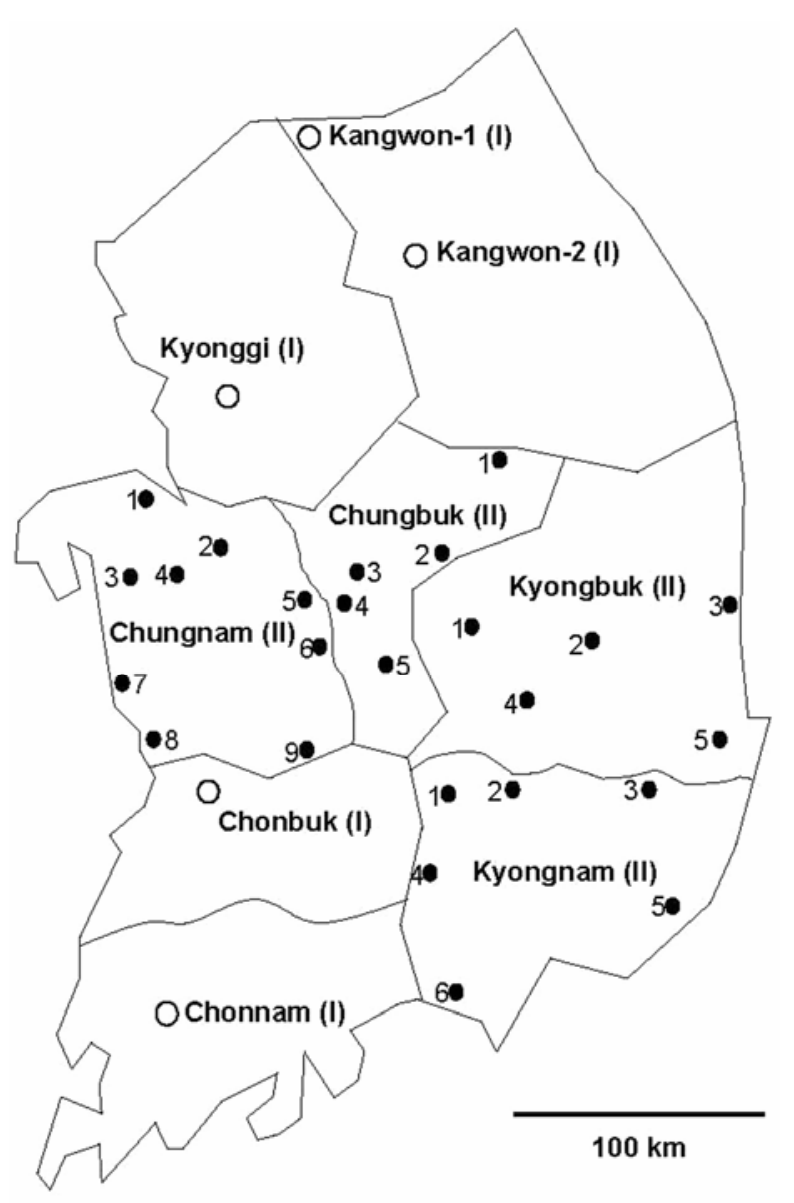

Fig. 1. Geographic locations of rice fields where Magnaporthe oryzae was sampled. We sampled group I isolates from one field in each of the three provinces in Korea (Chonnam, Chonbuk, and Kyonggi); two fields were sampled in the province of Kangwon province (open circles). Group I isolates of $M$. oryzae were sampled from two to six cultivars at each location, out of nine cultivars common in Korea (Table 1). Group II isolates were sampled from 26 blast scouting plots in Kyongnam, Kyongbuk, and Chungnam, and Chungbuk provinces (smaller solid dots). We collected group II isolates from a large number of cultivars as part of a pathotype survey conducted by the Rural Development Administration of Korea. 
blast-scouting plots (Fig. 1); however, the cultivar of origin is not known for most isolates. These cultivars included advanced breeding lines and a diverse group of cultivars, most of which are not used commonly in commercial cultivation. There was only one cultivar (Daesan) that overlapped between the groups. Because of the two different sampling strategies, subpopulations from the two groups were analyzed separately.

Isolation methods for the two groups were done as follows. For group I, single-conidial isolates were obtained from sporulating lesions after leaves were surface sterilized and placed on water agar. Only one monoconidial isolate was obtained from each plant. For long-term storage, all isolates were grown on oatmeal agar for 2 weeks at $23^{\circ} \mathrm{C}$, dried along with the medium, and kept in plastic cryo-storage tubes at $-20^{\circ} \mathrm{C}$. For group II, hyphal-tip isolates were obtained from single conidia germinating on agar medium as described previously (9). For long-term storage of group II isolates, each isolate was grown for 15 days on filter paper placed on potato dextrose agar. Filter papers were removed, placed in sterile envelopes, dried at room temperature, and stored at $-20^{\circ} \mathrm{C}$.

DNA fingerprinting and RFLPs. Culturing, DNA extraction, and DNA fingerprinting with restriction fragment length polymorphism (RFLP) probes MGR586 and MAGGY were done exactly as previously described (16). These two probes are moderately repetitive in $M$. oryzae and each one hybridizes to approximately 50 to 60 EcoRI restriction fragments from each isolate to produce highly variable DNA fingerprints $(1,5,8,13,14)$. In addition to fingerprinting, we determined RFLPs using several other probes using the same Southern blots and hybridization conditions described previously (16). First, we used a 1.2-kb KpnI-SalI fragment of pSK51 carrying the TLH1 gene, a member of a telomere-associated helicase gene family (6). Members of this gene family (up to 10 per strain) are ubiquitous among $M$. oryzae isolates from rice and seem to have undergone frequent deletion and amplification events. Second, we probed with a 1-kb HpaI fragment carrying the PWL2 host specificity gene isolated from pCB791 (21). Probed blots were stripped and rehybridized with other probes. Finally, we used mating-typespecific probes isolated from pSK43 and pSK44 (11) to determine mating type.

DNA fingerprints were scored from autoradiograms and analyzed as described previously (16). Briefly, we used a binary scoring system ( 1 for presence and 0 for absence at each band position) for each fingerprint and calculated fingerprint similarities using Nei and Li's index (15) (also known as the Dice coefficient): $S_{x y}=2 N_{x y} /\left(N_{x}+N_{y}\right)$, where $N_{x}$ and $N_{y}$ are the number of fragments in isolates $X$ and $Y$, respectively, and $N_{x y}$ is the number of fragments shared by $X$ and $Y$. The similarity matrix was used to construct a phenogram via the unweighted pair group method using arithmetic means (UPGMA) in the computer program NTSYS-pc version 2.2 (Exeter Software, Setauket, NY). Bootstrapping using 200 replications was done to assess the robustness of clusters in the phenograms using NTSYS-pc. Haplotype diversity of $M$. oryzae subpopulations was measured as $H^{\prime}=-\Sigma_{i}\left[p_{i} \cdot \ln \left(p_{i}\right)\right]$, where $p_{i}$ is the frequency of the $i$ th haplotype (7).
Analysis of population subdivision. To test for population subdivision, we conducted analyses of molecular variance (AMOVA) on fingerprint and RFLP data using the program Arlequin 2.0 (Genetics and Biometry Laboratory, Department of Anthropology, University of Geneva, Switzerland). We used AMOVA to estimate genetic differentiation $\left(\Phi_{\mathrm{ST}}\right)$ between isolate groups I and II, which confound locations and cultivars. Within Group I, we estimated $\Phi_{\mathrm{ST}}$ among cultivars within each location and among locations for each cultivar. Significance testing for $\Phi_{\text {ST }}$ was done using 1,023 permutations in Arlequin. Similar tests were not done for group II isolates because too few isolates were collected from each location and the cultivar of origin was not known for most isolates.

\section{RESULTS}

Diversity and fingerprint lineage structure. Among the 254 M. oryzae isolates from eight provinces of Korea (Fig. 1) in 1999, nearly every isolate had a unique fingerprint haplotype. We found 230 haplotypes with MGR586 and 237 haplotypes with MAGGY (Table 2). Estimates of haplotype diversity were nearly 1.0 in each of the nine locations (data not shown). Fingerprint similarities among all isolates was high for both markers, with similarities greater than 76 and $85 \%$ for MGR586 and MAGGY, respectively. Individual UPGMA phenograms derived from MGR586 and MAGGY showed similar topologies (data not shown); therefore, we combined both data sets. Haplotypes of 1999 isolates clustered into two distinct lineages, correlating perfectly with group I and group II from the different sets of host cultivars and locations (Fig. 2). The two lineages were supported by modest bootstrap values (74 and $77 \%$, respectively), with $71 \%$ similarity between them; similarity within each lineage was greater than $76 \%$.

We detected two to four EcoRI fragments in each isolate using TLH1 as a probe, resulting in a total of 17 haplotypes among the 254 isolates (Fig. 3). The haplotype diversity for TLH1 was slightly higher in group II than in group I even though the sample size was larger in group I (Table 2). The $P W L 2$ probe hybridized to one to three EcoRI fragments in each isolate, ranging in size from 0.8 to $20 \mathrm{~kb}$, resulting in 12 haplotypes (Fig. 3). As for $P W L 2$, haplotype diversity was slightly higher in group II than in group I (Table 2).

We compared multilocus haplotypes from the 1999 samples with those sampled by RDA from 1984 to 1999 (16); the latter sample was collected using the same sampling strategy for pathotype surveys as used for group II. Fingerprint haplotypes for group II isolates collected in 1999 clustered together with historical isolates collected by RDA (Fig. 4). In contrast, group I isolates formed a separate subgroup containing no historical isolates. The cluster with group I isolates had bootstrap support of $68.5 \%$, while there was no support for the cluster with group II isolates.

Population subdivision. As described above, MGR586 and MAGGY fingerprints revealed two distinct lineages that correlated to group I and group II isolates, sampled from disjunct sets of rice cultivars in different provinces. Moreover, groups I and II

TABLE 1. Sample sizes for group I isolates of Magnaporthe oryzae collected from each rice cultivar in four provinces of Korea in 1999

\begin{tabular}{|c|c|c|c|c|c|c|c|c|c|c|}
\hline \multirow[b]{2}{*}{ Province } & \multirow[b]{2}{*}{ Total no. of isolates } & \multicolumn{9}{|c|}{ Cultivars $^{\mathrm{a}}$} \\
\hline & & A & $\mathrm{B}$ & $\mathrm{C}$ & $\mathrm{D}$ & $\mathrm{E}$ & $\mathrm{F}$ & $\mathrm{G}$ & $\mathrm{H}$ & I \\
\hline Chonnam & 59 & 21 & 16 & - & - & - & 22 & - & - & - \\
\hline Chonbuk & 15 & 9 & 6 & - & - & - & - & - & - & - \\
\hline Kyonggi & 77 & 19 & 12 & 11 & 15 & 17 & - & 3 & - & - \\
\hline Kangwon-1 & 16 & - & - & 11 & - & - & - & - & 5 & - \\
\hline Kangwon-2 & 13 & 11 & - & - & - & - & - & - & - & 2 \\
\hline Total & 180 & 60 & 34 & 22 & 15 & 17 & 22 & 3 & 5 & 2 \\
\hline
\end{tabular}

a A: cv. Ilpum, B: cv. Hwaseong, C: cv. Chuchung, D: cv. Jinmmy, E: cv. Chukwang, F: cv. Daesan, G: cv. Bongkwang, H: cv. Odae, I: cv. Yumyung. 
each had several TLH1 and PLW2 haplotypes that were not found in the other group. AMOVA was performed as a statistical test for genetic differentiation between groups I and II. Genetic differentiation was significant $(P<0.03)$ for all probes (Table 3$)$. Estimates of $\Phi_{\mathrm{ST}}$, which can be interpreted as variation attributed to differentiation among subpopulations, were 36.5, 26.8, 3.3, and $1.8 \%$ of the total genetic variation with markers MGR586, MAGGY, TLH1, and PWL2, respectively, when all group I and II subpopulations were combined. Therefore, we found significant genetic differentiation of the $M$. oryzae subpopulations with respect to both geographic location and cultivar of origin, which were confounded in our sampling.

AMOVA was also performed among subpopulations within group I because sample sizes were large enough for some cultivars to test for differentiation while holding either cultivar or location constant (Table 3). When group I isolates from the same cultivars were analyzed separately, we found significant $(P<$ $0.05)$ differentiation among locations for most markers, with the exceptions of PWL2 for cvs. Ilpum and Chuchung (Table 3). Similarly, we found significant differentiation of group I isolates among cultivars within locations for most, but not all tests (Table 3).

Mating type of M. oryzae in Korea. To evaluate the potential for sexual recombination, we determined the mating type of all isolates by probing genomic DNA from each isolate with matingtype genes. All isolates hybridized to Mat1-1; none hybridized to Mat1-2.

\section{DISCUSSION}

The objectives of this study were to test for genetic differentiation among $M$. oryzae subpopulations in eight provinces in Korea and on different rice cultivars. Sampling large numbers of isolates during a single year allowed us to address population structure questions in more detail, complementing the long-term study in which we analyzed relatively small samples in each of almost 20 years (16). Results from the current study differ markedly from our previous study in two ways. First, we found two clusters of fingerprint haplotypes in 1999 with moderate bootstrap support that could be interpreted as lineages, whereas previously we did not detect any clear lineage structure. Second, we found strong evidence for genetic differentiation of M. oryzae subpopulations among group I haplotypes from different cultivars, whereas previously we found no correlation between cultivars and fingerprints. Furthermore, unlike most studies of

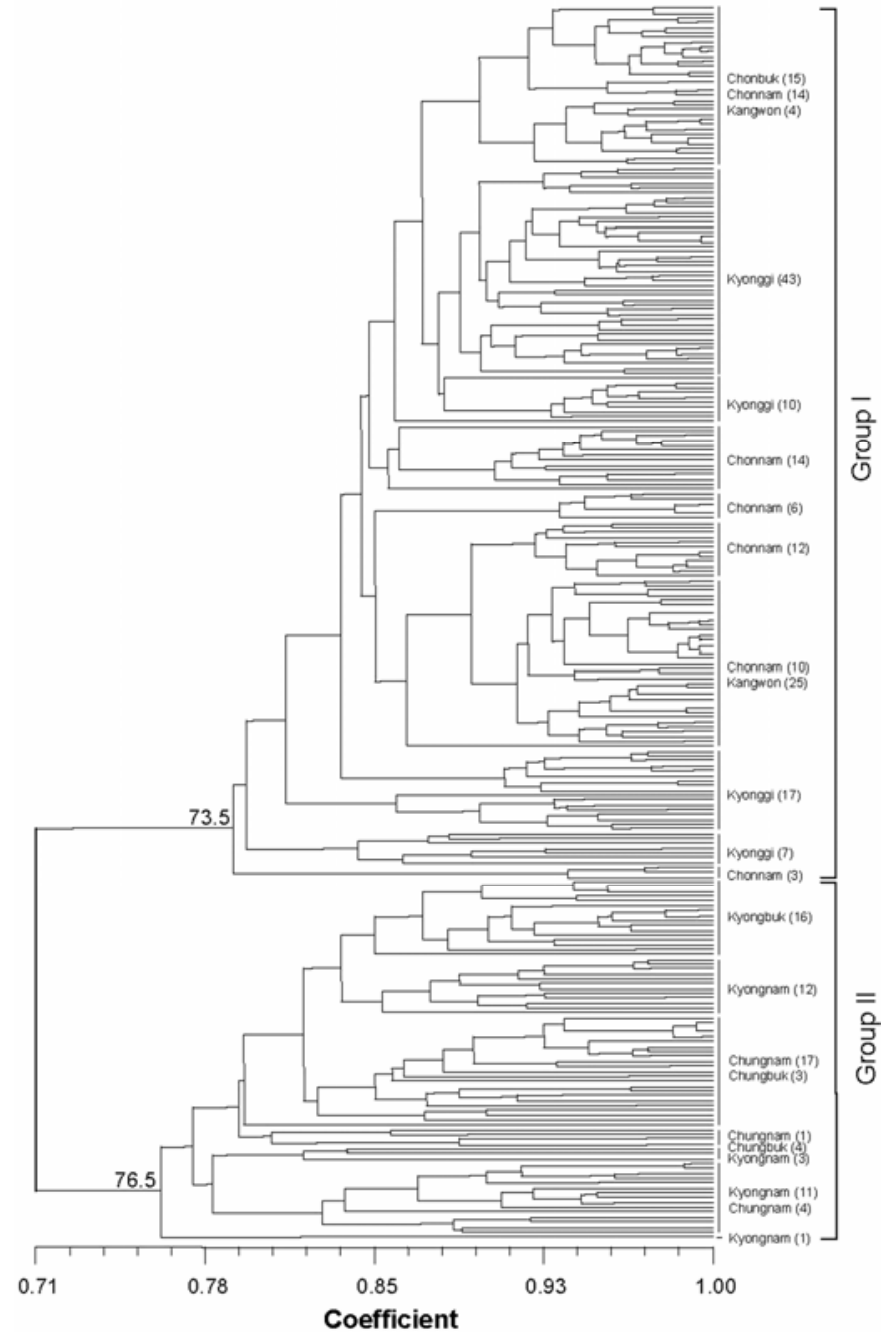

Fig. 2. Unweighted pair-group method with arithmetic average (UPGMA) phenogram of combined MGR586 and MAGGY fingerprint haplotypes of 254 Magnaporthe oryzae isolates collected in 1999. Group I isolates were collected from common cultivars in commercial fields in four provinces in Korea (Fig. 1), whereas group II isolates were sampled from rice blast scouting plots as part of a pathotype survey. Bootstrap values $>50 \%$ are shown for internal branches only. Vertical lines to the right indicate subgroups within group I and II with similarities between 81 and $94 \%$, which were arbitrarily defined to illustrate the province and number of isolates (in parentheses) from each location.

TABLE 2. Haplotype diversity of Magnaporthe oryzae subpopulations in Korea in 1999 based on restriction fragment length polymorphism (RFLP) fingerprinting (MGR586 and MAGGY) and RFLPs of two gene families (TLH1 and PWL2)

\begin{tabular}{|c|c|c|c|c|c|c|c|c|}
\hline \multirow[b]{2}{*}{ Province } & \multirow[b]{2}{*}{ No. of isolates } & \multirow{2}{*}{$\begin{array}{c}\text { No. of } \\
\text { cultivars }^{\mathrm{a}}\end{array}$} & \multirow{2}{*}{$\begin{array}{c}\text { MGR586 } \\
\text { haplotypes }^{\text {b }}\end{array}$} & \multirow{2}{*}{$\begin{array}{l}\text { MAGGY } \\
\text { haplotypes }\end{array}$} & \multicolumn{2}{|c|}{ TLH1 } & \multicolumn{2}{|c|}{$P W L 2$} \\
\hline & & & & & Haplotypes & Diversity $^{c}$ & Haplotypes & Diversity \\
\hline \multicolumn{9}{|l|}{ Group I } \\
\hline Chonnam & 59 & 3 & 53 & 59 & 7 & 0.639 & 7 & 0.672 \\
\hline Chonbuk & 15 & 2 & 13 & 14 & 3 & 0.257 & 1 & 0 \\
\hline Kyonggi & 77 & 6 & 72 & 75 & 5 & 0.413 & 5 & 0.462 \\
\hline Kangwon-1 & 16 & 2 & 14 & 13 & 3 & 0.658 & 3 & 0.667 \\
\hline Kangwon-2 & 13 & 2 & 7 & 11 & 2 & 0.282 & 2 & 0.282 \\
\hline Total & 180 & 9 & 159 & 172 & 9 & 0.550 & 8 & 0.519 \\
\hline \multicolumn{9}{|l|}{ Group II } \\
\hline Kyongnam & 27 & $>17$ & 26 & 23 & 9 & 0.650 & 7 & 0.707 \\
\hline Kyongbuk & 16 & $>12$ & 15 & 15 & 4 & 0.692 & 4 & 0.742 \\
\hline Chungnam & 22 & $>3$ & 22 & 21 & 8 & 0.758 & 4 & 0.697 \\
\hline Chungbuk & 9 & $>4$ & 9 & 9 & 6 & 0.889 & 3 & 0.556 \\
\hline Total & 74 & - & 72 & 68 & 14 & 0.823 & 8 & 0.745 \\
\hline Total & 254 & $>26$ & 231 & 240 & 17 & 0.685 & 12 & 0.600 \\
\hline
\end{tabular}

a Number of cultivars from which isolates were collected. Total number of cultivars for group II is not known.

${ }^{b}$ Number of isolates with distinct hybridizing banding patterns.

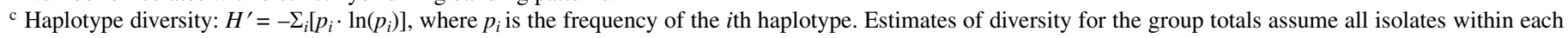
group were sampled from the same subpopulation. Similarly, total diversity pools all isolates from groups I and II. 
M. oryzae in other areas $(10,12,17,22)$, we found evidence of genetic differentiation of subpopulations on the same cultivars among locations, suggesting some degree of restricted gene flow within Korea not only between cultivars but between geographic areas.

The two distinct haplotype clusters in 1999 (Fig. 2) correlated to isolation from different sets of cultivars. When these same haplotypes were analyzed in relation to historical collections of $M$. oryzae (16) the lineage structure is not as evident, although group I haplotypes still formed a distinct cluster with moderate bootstrap support (Fig. 4). Group II isolates were collected from blast scouting plots with a high diversity of rice cultivars for detecting novel pathotypes; historical isolates analyzed in the previous study were collected in exactly the same way. Curiously, sampling from these scouting plots appears to be inappropriate for assessing the population of $M$. oryzae that exists on the cultivars that are planted in the majority of commercial rice fields in Korea. Populations of $M$. oryzae from rice-production fields (group I) are genetically distinct, forming a fingerprint cluster distinct from the isolates collected from scouting plots for pathotype surveys. Whether we consider these two clusters as separate fingerprint lineages is a matter of semantics. Genetic similarity may not be as high within lineages defined in other studies using MGR fingerprints only $(13,25)$, nor as dissimilar between lineages, but nonetheless these putative lineages appear to have a strong biological basis because they correlate perfectly to group I and II isolates from the different sets of cultivars. If group I is considered to form a distinct lineage, we might conclude that the $M$. oryzae population in Korea comprises several additional fingerprint lineages (Fig. 4), although no biological differences are known among them, and they lack bootstrap support.

\section{TLH1 Type}

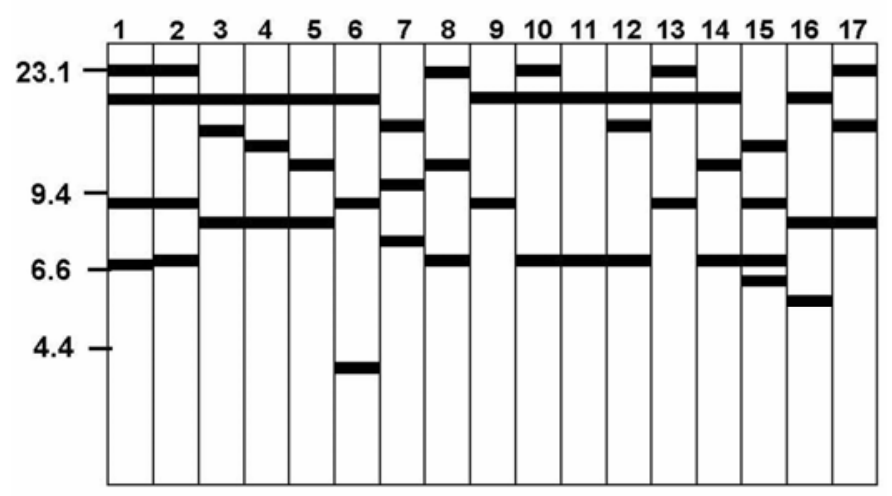

PWL2 Type

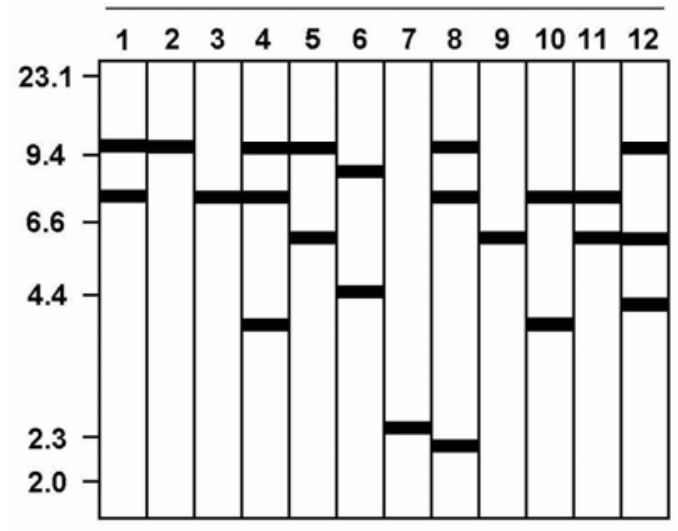

Fig. 3. Schematic diagrams of unique restriction fragment length polymorphism haplotypes among 254 Magnaporthe oryzae isolates detected by using $T L H 1$ or $P W L 2$ as probes.
The correlation of group I and group II isolates in 1999 with different lineages could be caused by either selection for host specialization or differentiation owing to restricted migration. These isolate groups were sampled from distinct sets of cultivars in different geographic locations, therefore cultivars and locations are confounded. To determine which of these factors is responsible for this differentiation, $M$. oryzae would need to be sampled from both sets of cultivars in close proximity, in replicate locations, and then genotyped. Unfortunately, this was not done for this study because we did not expect to find such marked differentiation. Although group I cultivars are commonly grown throughout Korea, disease incidence on them was too low in four

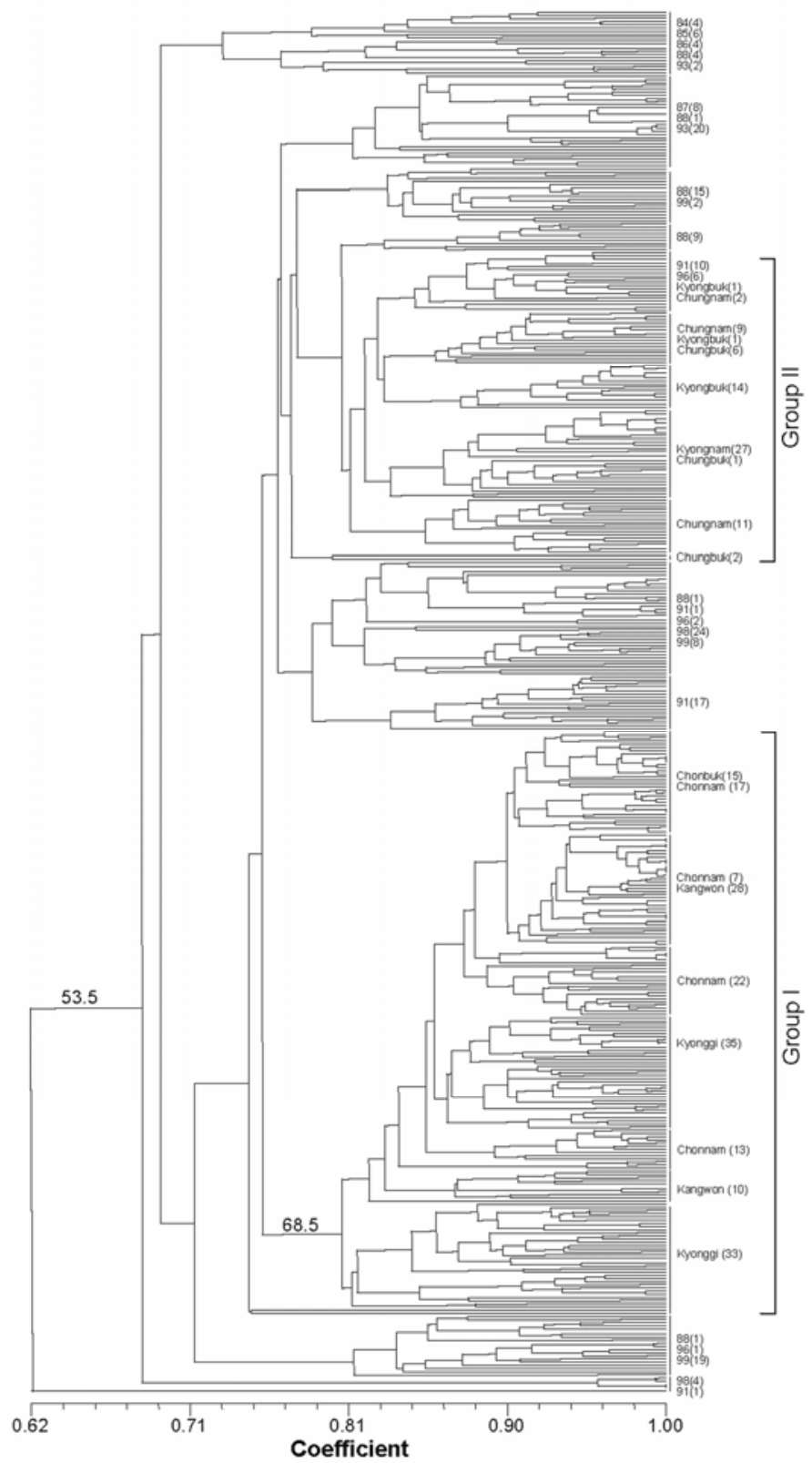

Fig. 4. Unweighted pair-group method with arithmetic average (UPGMA) phenogram of combined MGR586 and MAGGY fingerprint haplotypes for 254 Magnaporthe oryzae isolates collected in 1999 and 176 isolates collected from 1984 to 1999 (data from Park et al. [16]). Group I isolates were collected from common cultivars in commercial fields in four provinces in Korea (Fig. 1 ), whereas all other isolates were sampled from rice blast scouting plots as part of a pathotype survey. Bootstrap values $>50 \%$ are shown for internal branches only. Vertical lines to the right indicate subgroups with similarities between 74 and $91 \%$, which were arbitrarily defined to illustrate the year (for historical samples) or province (for 1999 samples from this study) and number of isolates (in parentheses). 
provinces at the time of sampling to obtain reasonable sample sizes. This difference in disease incidence could have been caused by genetic differentiation of the $M$. oryzae population in different locations, but it could also have been caused by a number of other factors, for example, environmental conditions. To avoid the confounding factors, we analyzed differentiation for group I haplotypes among locations within the same cultivars, and among cultivars within locations. Almost all tests, for all markers, showed significant differentiation among locations (within cultivars) and among hosts (within locations). Therefore, contrary to our interpretation of data from the historical samples (16), we found evidence of cultivar specialization within a single year of sampling on commercial cultivars.

In contrast to other studies on population structure of $M$. oryzae $(10,12,17,22)$, we found significant genetic differentiation among isolates from the same cultivars collected from different locations within a relatively small area. The maximum distance between collection sites of group I isolates in Korea was $362 \mathrm{~km}$. This scale is roughly comparable to studies done in Georgia, United States (22), Italy (17), or Iran (10), where little or no differentiation was found. Moderate levels of genetic differentiation were found between upland and lowland sites in the Philippines (1), but large ecological differences between sites were confounded with location and restricted migration could not be separated from selection as a cause of differentiation. Evidence for differentiation on a small geographic scale, however, was found by Kumar et al. (12) who sampled up to 10 lineages in one population and different lineages (and less diversity) in another location only 4 to $7 \mathrm{~km}$ away. An important distinction between south and east Asian populations and the other locations, is the length of time rice cultivation and $M$. oryzae have been present. Older populations may have persisted long enough to allow $M$. oryzae populations to diverge over time, whereas younger populations may still show signs of historical gene flow (20).

Results from this study of a broad geographic sampling in a single year in Korea lead to two interesting interpretations that contradict our previous findings from a 20-year study (16). First, genetic differentiation among group I isolates from different cultivars in the same location represents a novel finding for $M$. oryzae in Korea. The simplest explanation for this differentiation is that selection has resulted in host specialization. However, no pathotype data are available for group I isolates in this study and, therefore, we cannot dissect this relationship further.

Second, we proposed that finding novel haplotypes each year in a multi-year study (16) could be explained by sexual reproduction and periodic bottlenecks. In this study, we found only one mating type, Mat1-1, among M. oryzae isolates, ruling out sexual reproduction as a source of novel haplotypes. Furthermore, we reject the bottleneck hypothesis for the group I subpopulation. We previously hypothesized that populations undergoing periodic bottlenecks with relatively few fingerprint haplotypes surviving between cropping seasons would have little or no geographic structure because $M$. oryzae would spread across the rice-growing areas of Korea each year. The finding of geographic differentiation among group I isolates is not consistent with this hypothesis.

An additional reason to reject the bottleneck hypothesis is because of the variation observed within both lineages from larger samples in a single year. Lineages of $M$. oryzae are often defined using arbitrary criteria based on percent similarity of fingerprint haplotypes; the typical definition is $<80 \%$ similarity between lineages and $>90 \%$ similarity within lineages $(13,25)$. Although these criteria are arbitrary, the discrete clustering of haplotypes in phenograms (based on similarity matrices) are consistent with this type of definition. The lineages we found in Korea are not as distinct as those defined in other studies, but they do correlate to distinct groups of isolates, and therefore, we interpret them as being biologically and evolutionarily independent lineages. The question arises, however, as to why lineages in Korea are not as discrete as in other populations. Variation within a lineage may depend on population size and its persistence over time. A lineage with lower similarities among haplotypes, such as observed in Korea, is more likely to evolve in a large population persisting over a long period of time, allowing mutations to accumulate. In contrast, if entire lineages experience extinction and recolonization, then variation within lineages at any given time is likely to be small because less time may have elapsed for mutations to accumulate. Therefore, our previous hypothesis that Korean populations have undergone repeated bottlenecks is not supported by results of this study.

The contrast between the two studies of $M$. oryzae population structure in Korea, from a single year (this study) and a 20-year period (16), provides complementary and somewhat contradictory interpretations of the evolution of this fungus. However, these differences can be resolved by considering the differences in sampling, both in terms of the cultivars sampled and the sample sizes analyzed. Group II isolates in this study were collected from scouting plots (blast nurseries) using identical methods as for the historical samples, and they are also in the same fingerprint lineage. Group I isolates were collected from commercial rice fields and form a distinct fingerprint lineage. The turnover in fingerprinting haplotypes in the historical study might be either an artifact of sampling of a relatively small number of isolates each

TABLE 3. Analysis of molecular variance (AMOVA) on subpopulations of Magnaporthe oryzae to test for genetic differentiation between isolate groups I and II, group I isolates among location (within cultivars) and group I isolates among cultivars (within locations)

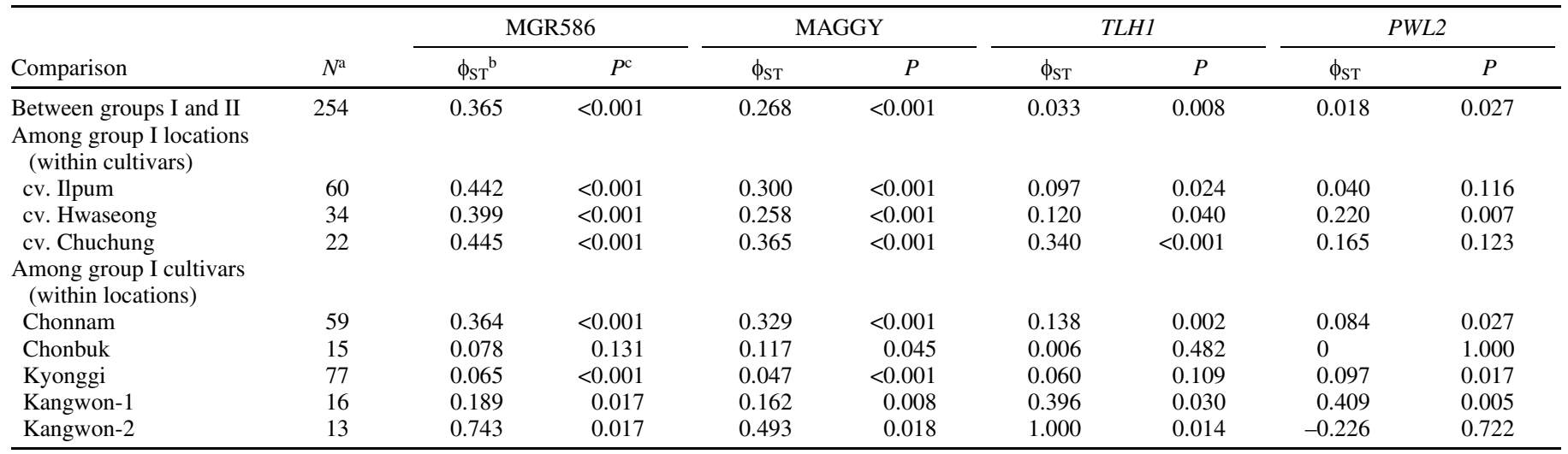

\footnotetext{
${ }^{a}$ Number of isolates.

${ }^{\mathrm{b}} \phi_{\mathrm{ST}}$ is the proportion of genetic variation due to differences among subpopulations: $\phi_{\mathrm{ST}}=\sigma^{2}{ }_{\mathrm{a}} / \sigma^{2} \mathrm{~T}$, where $\sigma_{\mathrm{a}}^{2}$ is variation among subpopulations and $\sigma^{2}{ }_{\mathrm{T}}$ is variation among all individuals, independent of subpopulations.

${ }^{c} P$ values were estimated based on 1,023 permutations.
} 
year, or a real turnover of haplotypes from small populations of $M$. oryzae from scouting plots. Sampling from scouting plots was originally done for detecting novel pathotypes, which cannot be done on large samples because of the resources required, and therefore, only small numbers of isolates were collected and analyzed from each province each year. However, samples from scouting plots did not capture a random sample of the $M$. oryzae population from commercial fields; scouting plots contain cultivars that were chosen for monitoring changes in pathotypes or for finding new pathotypes. In fact, the differentiation of distinct $M$. oryzae lineages from scouting plots and commercial fields indicates that one sample is a very poor predictor of the other. Future studies of this and other plant pathogenic fungi, therefore, need to be based on samples to address specific questions, and caution must be taken in interpreting results from collections made for other purposes.

\section{ACKNOWLEDGMENTS}

This research was partially supported by a grant from Biogreen 21 project (20050401034629) funded by Rural Development Administration, by a grant from Crop Functional Genomics Center (CG1141) of the 21st Century Frontier Research Program funded by the Ministry of Science and Technology, and by Korean Research Foundation Grant (KRF-2006005-J04701) to Y. H. Lee. S.-Y. Park thanks the Ministry of Education for providing a graduate fellowship through the BK21 program.

\section{LITERATURE CITED}

1. Chen, D., Zeigler, R. S., Leung, H., and Nelson, R. J. 1995. Population structure of Pyricularia grisea at two screening sites in the Philippines. Phytopathology 85:1011-1020.

2. Couch, B. C., Fudal, I., Lebrun, M.-H., Tharreau, D., Valent, B., van Kim, P., Notteghem, J. L., and Kohn, L. M. 2005. Origins of host-specific populations of the rice blast fungus Magnaporthe oryzae in crop domestication with subsequent expansion of pandemic clones on rice and weeds of rice. Genetics 170:613-630.

3. Couch, B. C., and Kohn, L. M. 2002. A multilocus gene genealogy concordant with host preference indicates segregation of a new species, Magnaporthe oryzae, from M. grisea. Mycologia 94:683-693.

4. Don, L. D., Kusaba, M., Urashima, A. S., Tosa, Y., Nakayashiki, H., and Mayama, S. 1999. Population structure of the rice blast fungus in Japan examined by DNA fingerprinting. Ann. Phytopathol. Soc. Jpn. 65:15-24.

5. Farman, M. L., Taura, S., and Leong, S. A. 1996. The Magnaporhte grisea DNA fingerprinting probe MGR586 contains the $3^{\prime}$ end of an inverted repeat transposon. Mol. Gen. Genet. 251:675-681.

6. Gao, W., Khang, C. H., Park, S.-Y., Lee, Y.-H., and Kang, S. 2002. Evolution and organization of a highly dynamic, subtelomeric helicase gene family in the rice blast fungus Magnaporthe grisea. Genetics $162: 103-112$

7. Grünwald, N. J., Goodwin, S. B., Milgroom, M. G., and Fry, W. E. 2003. Analysis of genotypic diversity data for populations of microorganisms. Phytopathology 93:738-746.
8. Hamer, J. E., Farrall, L., Orbach, M., Valent, B., and Chumley, F. G. 1989. Host species-specific conservation of a family of repeated DNA sequences in the genome of a fungal plant pathogen. Proc. Natl. Acad. Sci. USA 86:9981-9985.

9. Han, S.-S. 1996. Genetic variability and population structure of Korean isolates of Pyricularia grisea. Ph.D. Diss. Tokyo University of Agriculture, Japan.

10. Javan-Nikkhah, M., McDonald, B. A., Banke, S., and Hedjaroude, G.-A. 2004. Genetic structure of Iranian Pyricularia grisea population based on rep-PCR fingerprinting. Eur. J. Plant Pathol. 110:909-919.

11. Kang, S., Chumley, F. G., and Valent, B. 1994. Isolation of the matingtype genes of the phytopathogenic fungus Magnaporthe grisea using genomic subtraction. Genetics 138:289-296.

12. Kumar, J., Nelson, R. J., and Zeigler, R. S. 1999. Population structure and dynamics of Magnaporthe grisea in the Indian Himalayas. Genetics 152:971-984.

13. Levy, M., Correa-Victoria, F. J., Zeigler, R. S., Hu, S., and Hamer, J. E. 1993. Genetic diversity of the rice blast fungus in a disease nursery in Colombia. Phytopathology 83:1427-1433.

14. Levy, M., Romao, J., Marchetti, M. A., and Hamer, J. E. 1991. DNA fingerprinting with a dispersed repeated sequence resolves pathotype diversity in the rice blast fungus. Plant Cell 1991:95-102.

15. Nei, M., and Li, W.-H. 1979. Mathematical model for studying genetic variation in terms of restriction endonucleases. Proc. Natl. Acad. Sci. USA 76:5269-5273.

16. Park, S.-Y., Milgroom, M. G., Han, S. S., Kang, S., and Lee, Y.-H. 2003. Diversity of pathotypes and DNA fingerprint haplotypes in populations of Magnaporthe grisea in Korea over two decades. Phytopathology 93:13781385.

17. Piotti, E., Rigano, M. M., Rodino, D., Rodolfi, M., Castiglione, S., Picco, A. M., and Sala, F. 2005. Genetic structure of Pyricularia grisea (Cooke) Sacc. isolates from Italian paddy fields. J. Phytopathol. 153:80-86.

18. Roumen, E., Levy, M., and Notteghem, J. L. 1997. Characterization of the European pathogen population of Magnaporthe grisea by DNA fingerprinting and pathotype analysis. Eur. J. Plant Pathol. 103:363-371.

19. Shen, Y., Zhu, P., Yuan, X., Zhao, X., Manry, J., Rojas, C., Shahjahan, A. K. M., and Levy, M. 1996. The genetic diversity and geographic distribution of Pyricularia grisea in China. Sci. Agric. Sinica 29:39-46.

20. Slatkin, M. 1987. Gene flow and the geographic structure of natural populations. Science 236:787-792.

21. Sweigard, J. A., Carroll, A. M., Kang, S., Farrall, L., Chumley, F. G., and Valent, B. 1995. Identification, cloning, and characterization of $P W L 2$, a gene for host species specificity in the rice blast fungus. Plant Cell 7:1221-1233.

22. Tredway, L. P., Stevenson, K. L., and Burpee, L. L. 2005. Genetic structure of Magnaporthe grisea populations associated with St. Augustinegrass and tall fescue in Georgia. Phytopathology 95:463-471.

23. Xia, J. Q., Correll, J. C., Lee, F. N., Marchetti, M. A., and Rhoads, D. D. 1993. DNA fingerprinting to examine microgeographic variation in the Magnaporthe grisea (Pyricularia grisea) population in two rice fields in Arkansas. Phytopathology 83:1029-1035.

24. Zeigler, R. S. 1998. Recombination in Magnaporthe grisea. Annu. Rev. Phytopathol. 36:249-275.

25. Zeigler, R. S., Cuoc, L. X., Scott, R. P., Bernardo, M. A., Chen, D. H., Valent, B., and Nelson, R. J. 1995. The relationship between lineage and virulence in Pyricularia grisea in the Philippines. Phytopathology 85:443-451. 